Rabaska

Revue d'ethnologie de l'Amérique française

\title{
Le Forum canadien de recherche publique sur le patrimoine
}

\section{Lucie K. Morisset}

Volume 6, 2008

URI : https://id.erudit.org/iderudit/019992ar

DOI : https://doi.org/10.7202/019992ar

Aller au sommaire du numéro

Éditeur(s)

Société québécoise d'ethnologie

ISSN

1703-7433 (imprimé)

1916-7350 (numérique)

Découvrir la revue

Citer ce document

Morisset, L. K. (2008). Le Forum canadien de recherche publique sur le patrimoine. Rabaska, 6, 123-133. https://doi.org/10.7202/019992ar d'utilisation que vous pouvez consulter en ligne.

https://apropos.erudit.org/fr/usagers/politique-dutilisation/ 


\title{
Le Forum canadien de recherche publique sur le patrimoine
}

\author{
LuCIE K. MorisseT \\ Université du Québec à Montréal
}

Le Forum canadien de recherche publique sur le patrimoine (FCRP) / Canadian Forum for Public Research on Heritage (CFPRH) est un réseau stratégique de recherche, d'observation, d'information et de concertation autour du paysage construit et des mémoires qu'il convoque. Mis sur pied en janvier 2006, il a reçu en 2008 l'aval du Conseil de recherches en sciences humaines du Canada qui lui a accordé, dans le cadre de son programme "Réseaux stratégiques de connaissance», une subvention septennale de deux millions de dollars. Après une première table ronde tenue à Charlottetown en 2006, le Forum a tenu une rencontre officielle de démarrage de ses travaux à Yellowknife, en juin 2008, en marge du congrès annuel de la Société pour l'étude de l'architecture au Canada; ses douze chercheurs principaux, ainsi qu'un représentant des partenaires du Forum, ont alors dressé les grandes lignes de la programmation des activités de maillage, de formation et de recherche visées par les objectifs du réseau. Il s'agit, a priori, de convoquer les forces vives du patrimoine, c'est-à-dire les chercheurs, les décideurs et les collectivités, à réfléchir à la patrimonialisation dans la société contemporaine ; pour ce faire, le Forum mise sur un noyau de douze chercheurs principaux - parmi lesquels six titulaires de Chaires de recherche du Canada -, auxquels s'associent six collaborateurs, professeurs et chercheurs universitaires canadiens ou étrangers, et onze partenaires, organismes de recherche, de diffusion, de réseautage ou de décision dans le domaine du patrimoine.

Par rapport aux initiatives existantes, dissoutes dans la «culture", atomisées par sujet, par discipline ou par région, ou qui dissocient la diffusion et la recherche, le FCRPP vise à recentrer, à désenclaver, à démocratiser et à redéployer le patrimoine comme objet de recherche scientifique et au sein de la société civile (qui en est le principal utilisateur). Cette problématique est transdisciplinaire et met en jeu la culture canadienne ; interpellées partout au Canada par la mobilité et la diversité grandissantes des populations, les mémoires patrimoniales fluctuantes - de moins en moins liées à un « héritage 
commun »-et les relations que les diverses collectivités entretiennent avec leur environnement, de même que la définition de l'attachement de ces collectivités à un « patrimoine » et la gestion de cet attachement constituent un ensemble de problèmes d'ordre structurel, persistant et prépondérant.

\section{Le patrimoine comme objet de recherche publique}

À l'instar du ministère du Patrimoine canadien, qui le définit ainsi pour énoncer sa mission, le Forum conçoit le patrimoine comme un outil de cohésion et de création. Si, dans leur large éventail, les enjeux pragmatiques que soulève le "patrimoine bâti », du fait de sa nature environnementale, occupent régulièrement l'avant-plan de l'actualité, l'importance du paysage construit ainsi désigné dans la formation des identités collectives a été aussi largement démontrée. Or, si chercheurs et acteurs ne communiquent pas et que les constructions patrimoniales restent isolées, comment peut-on aspirer à quelque identité canadienne et comment, qu'une telle identité soit ou non souhaitable, faire en sorte que le patrimoine soit effectivement reconnu et approprié par les communautés auxquelles on le crédite ? En effet, on observe que le patrimoine, jadis l'instrument des constructions politiques nationales, relève dorénavant des collectivités proches qui s'y représentent. Or, ce « patrimoine de proximité », qui, de pair avec le «tout-patrimoine», fragmente l'investissement affectif des populations disséminées au Canada, soulève un problème considérable, auquel seul le réseautage peut tenter de répondre : en effet, personne ne sait comment accompagner ce mode quasi individuel de représentation collective, ni sur le plan des constructions identitaires, ni sur celui de l'administration quotidienne de ces représentations dans notre environnement quotidien. Ainsi, bien que les choix et la logistique, hérités des époques qui nous ont précédés, viennent pour l'instant «d'en haut» (de scientifiques et de structures nationales, provinciales, parfois régionales), l'affection patrimoniale, elle, devrait dorénavant naître « d'en bas »-ne serait-ce que pour justifier, dans une société démocratique, l'investissement public en faveur du patrimoine. En d'autres mots, si tant est qu'il doive y avoir identités collectives, il importe d'imaginer des structures de sélection et de valorisation patrimoniales plus adéquates par rapport au mode contemporain d'appropriation de l'environnement et une mise en réseau des compétences qui permettra, ici de comprendre la valeur publique du patrimoine, là de partager une solution novatrice de conservation.

C'est pourquoi le FCRPP vise à mobiliser décideurs et chercheurs de tous milieux dans la conception d'un dialogue entre l'expertise (qui soutient les choix du patrimoine) et la proximité (qui le vit) plus propice à susciter et à encadrer l'affection collective envers un patrimoine viable et pertinent. Il s'agit de rendre les résultats de la recherche disponibles à la société (dans sa 
diversité); mais cela requiert aussi l'intégration des recherches sur le patrimoine au Canada (caractérisation, gestion, valorisation) et de celles sur les représentations culturelles et leur formation (expérience et médiation), de la décision et de l'utilisation/demande du patrimoine (par le public). Le FCRPP compte donc faire du patrimoine un objet de recherche publique, et non plus seulement, soit un objet approprié par des groupes associatifs souvent dépourvus de moyens scientifiques, soit un instrument abstrait porté par quelque institution nationale. En d'autres mots, en vue de résoudre les problématiques patrimoniales contemporaines et, ce faisant, contribuer à l'évolution des pratiques et de la société en redéfinissant l'utilisateur/ demandeur (le citoyen) au centre des politiques du patrimoine et en apportant des solutions de gestion au parc patrimonial, le Forum aspire à constituer le patrimoine en un sujet de recherche collaborative de haut niveau et allant des spéculations épistémologiques jusqu'à l'intervention pragmatique, mais dont les visées restent ancrées aux besoins des communautés d'aujourd'hui du fait du maillage constant, soutenu par le réseau, entre l'université et le monde qui l'entoure.

\section{Un réseau, trois chantiers, plusieurs secteurs et de multiples disciplines}

La mission du FCRPP est de parvenir à un maillage qui optimise les activités de recherche en patrimoine, tant au plan de la décision que de la diffusion, en articulant le réseautage autour de problèmes pancanadiens, afin de comprendre et d'évaluer les conditions de réalisation de "patrimoines de proximité » fédérateurs et représentatifs des aspirations de la société civile. On mise donc sur la concertation des acteurs de la recherche, de la décision et de l'utilisation autour, pour l'instant, de trois grands « chantiers » qui convoquent les forces vives locales et nationales et qui, du fait de l'expertise constituée et de la spécificité des cas, pourraient distinguer la contribution scientifique canadienne sur la scène internationale. Premièrement, le "patrimoine religieux », qui inclut les couvents et les églises des traditions religieuses historiques, dorénavant menacés de désaffectation et dont le péril a fait la manchette, de Victoria à Saint-Jean (T.-N.), rassemble aussi, par exemple, des recherches sur le patrimoine immatériel et le patrimoine spirituel autochtone. Deuxièmement, le « patrimoine moderne », bien caractéristique du territoire pancanadien, permet d'aborder, outre les questions relatives aux interrelations entre le patrimoine et la modernité (les pratiques artistiques, l'identification du patrimoine bâti moderne, etc.), les problèmes spécifiques de conservation et de valorisation du «Canada Modern » qui interpellent l'ensemble des acteurs du patrimoine au Canada. Troisièmement, la « mémoire patrimoniale des Canadiens » désigne le double corpus des patrimoines et des patrimonialisations engendrés au fil de la construction canadienne (et 
des éventuelles « sub-constructions » provinciales ou régionales au Canada), précisément faite d'adaptations et de métissages de nature à enrichir la compréhension des mécanismes mémoriels et identitaires sous-jacents du patrimoine et de la narration patrimoniale ; ce chantier fédère les questions et les pratiques relatives aux institutions, aux lois, aux statuts, aux discours patrimoniaux et les recherches en matière de théorie du patrimoine.

Face à ces trois chantiers, le Canada dispose de forces majeures, qui nourrissent et nourriront sa contribution à la résolution des problèmes généraux et d'envergure mondiale ainsi découpés : en attestent, par exemple, les presque séculaires Commission des lieux et monuments historiques du Canada et agence gouvernementale Parcs Canada, la Fondation Héritage Canada, la Société pour l'étude de l'architecture au Canada, etc. En recherche, on peut dénombrer plusieurs percées significatives : pensons au système de qualification de valeurs patrimoniales, développé ici dès les années 1990 soit bien avant que le Getty Institute publie son modèle de gestion par les valeurs - ou à la longueur d'avance prise au départ du Québec dans la sauvegarde des églises, reconnue internationalement. Il faut dire que, en sus d'une méthodologie commune de caractérisation et de médiation, le Canada peut compter sur un atout majeur : la coexistence de deux univers « de fondation ", cristallisée dans la langue, mais aussi dans les institutions, les lois et les pratiques qui ancrent les rapports des collectivités à leur environnement. C'est sur cette unique perspective comparative, puissant levier pour la recherche canadienne en matière de patrimoine, que le FCRPP mise.

Réseau de réseaux et réseau d'acteurs individuels, le FCRPP aspire à optimiser les efforts de recherche des uns et des autres, d'une part pour résoudre les questions générales et spécifiques rassemblées à l'enseigne des trois chantiers désignés et celles qui seront mises au jour par la mise en commun des problématiques, d'autre part pour accroître l'attractivité canadienne en matière de formation à la recherche et les capacités de nos institutions à accueillir et à accompagner des nouveaux chercheurs en patrimoine mieux qualifiés.

Ainsi, le Forum, du fait de la rencontre des réseaux de ses membres et parce qu'il agit comme portail de la recherche, permet de constituer des équipes d'intervention pluridisciplinaires convoquant des expertises hautement spécialisées. En amont et en aval des projets de recherche ou d'intervention de ses membres, le Forum vise à permettre d'utiliser une force nationale pour résoudre des problèmes locaux, d'emprunter à des problématiques pour en nourrir d'autres (par exemple, la mémoire des communautés religieuses pourrait-elle reposer sur les moyens qui ont permis à des communautés autochtones de retrouver leur patrimoine " sacré »?) et d'aborder des questions de recherche pancanadiennes et multisectorielles, 
comme celles que nous avons énoncées, qu'aucun acteur, sous peine d'être écrasé par l'envergure du problème ou des partenariats requis, ne peut aborder dans l'isolement actuel. Les chantiers suscitent ainsi le regroupement de communautés en grappes et la construction de groupes opérationnels réunissant chercheurs et utilisateurs de divers milieux dans la résolution des problèmes (par exemple, l'avenir des centaines d'églises qui seront désaffectées dans les prochaines années) et dans la concrétisation de solutions nées du partage des expertises (par exemple, la prise en charge d'une église par une collectivité locale pour ses besoins communautaires).

Simultanément, tout en reconfigurant la recherche et l'expertise en abstrayant les barrières géographiques habituelles (particulièrement dans le domaine du patrimoine), la mise en réseau exercée par le Forum vise à accroître la capacité et la qualité de la formation en patrimoine. D'une part, seul un tel maillage pancanadien peut absorber la demande internationale en formation ; d'autre part, chacune de nos institutions, séparément, ne détient pas un spectre de spécialisations et de spécialistes assez large pour offrir un véritable cursus en patrimoine, ce que des parcours interuniversitaires incitant à la mobilité des étudiants pallieraient. Le Forum permet alors d'accroître la qualité de la formation en patrimoine en élargissant la gamme des domaines convoqués dans une seule formation transversale, en offrant une expérience pancanadienne plus pertinente et plus riche en cas et en perspectives apportés par les chercheurs et partenaires, en donnant accès aux meilleurs spécialistes selon les sujets adoptés et en mettant en partage des foyers d'accueil au profit des postdoctorants. Le réseautage du Forum pallie ainsi la fragmentation des moyens d'acquisition des savoirs en patrimoine, en permettant aux étudiants et aux jeunes chercheurs d'acquérir des compétences spécialisées de trois ordres. Premièrement, par des activités de formation, les jeunes praticiens et chercheurs pourront acquérir une vision pancanadienne (et comparative) du patrimoine et de ses problématiques; deuxièmement, grâce au spectre des spécialisations convoquées (études et recherches dans les différents foyers d'accueil), ils développeront une vision intégratrice du patrimoine, au-delà du regard issu d'une seule approche ou centré sur un seul type d'objet; troisièmement, grâce aux partenariats extrauniversitaires, étudiants, jeunes chercheurs et jeunes praticiens gagneront un sens de pertinence du patrimoine, comme un phénomène bien concret ancré aux besoins de la société. Ces compétences permettront, tantôt de mieux répondre aux demandes en recherche publique et des structures décisionnelles, tantôt de contribuer directement au renouveau des recherches en matière de patrimoine, selon leur niveau. Seul un réseau comme le Forum, du fait de la mise en commun des problématiques et des ressources, peut permettre à un étudiant de véritablement se spécialiser dans ce domaine, en complétant dans plusieurs 
sites $2^{\mathrm{e}}, 3^{\mathrm{e}}$ cycle et postdoctorat au départ de programmes de $1^{\mathrm{er}}$ cycle variés auxquels participent les chercheurs.

Enfin, multilatéralement, les activités du Forum donnent une nouvelle visibilité à la recherche sur le patrimoine, stimulant le renouveau des savoirs et l'intérêt au sein des communautés de recherche et de la société civile. Il s'agit, en somme, d'accompagner la naissance de patrimoines canadiens en mobilisant une recherche en mouvement, au sein d'un réseau grandissant, à son tour pépinière de chercheurs.

\section{L'économie et l'écologie du patrimoine pour/comme axes transversaux}

En intégrant les spéculations théoriques et pragmatiques sur le patrimoine et sur les représentations culturelles à la définition de pratiques et de recherches, les problématiques auxquelles aspire à répondre le FCRPP relèvent d'un double défi qui rend perméable les uns aux autres les trois chantiers qu'il traverse. Celui, inhérent au patrimoine, de la valorisation intégrée de celui-ci, au départ de sa caractérisation et incluant les enjeux matériels de sa mise en valeur : c'est « l'économie » du patrimoine. Puis, celui de la participation citoyenne à la patrimonialisation, seule garante de l'utilité sociale et culturelle d'un patrimoine définitoire d'identités : c'est « l'écologie » du patrimoine.

Le défi de « l'économie » du patrimoine (c'est-à-dire, étymologiquement, l'organisation et l'administration des divers éléments du patrimoine, si l'on conçoit celui-ci comme un système) mobilise les chercheurs et les décideurs, mais aussi les praticiens, sachant qu'une économie contextualisée du patrimoine implique aussi de considérer la patrimonialisation comme un levier de requalification (des villes, des villages, etc.) ; en effet, le patrimoine ne peut plus être pensé en seuls termes de conservation ou de muséification. « Construire » le patrimoine en édifiant le paysage de demain implique donc une réflexion qui englobe, à tous ses niveaux, les praticiens de la création et de la communication, en sus de ceux de la conservation et des décideurs usuels du patrimoine, que le Forum rejoint aussi à travers ses partenariats et les volets contextualisés des projets de recherche qu'il fédère. Quant à lui, le défi de «l'écologie » du patrimoine (les rapports internes entre le patrimoine et son milieu immédiat), qui sous-tend le déploiement de l'économie, repose sur une interaction dynamique (c'est-à-dire une interaction qui donne de véritables moyens d'expression à ses participants) entre l'expertise de tous niveaux, qui " choisit» le patrimoine, et les utilisateurs de celui-ci, en l'occurrence les praticiens, mais plus encore les citoyens qui vivent auprès $\mathrm{du}$ patrimoine et en interrelation avec lui : le patrimoine est leur environnement.

Ce double défi de l'économie et de l'écologie du patrimoine traverse la programmation du FCRPP et transparaît de chacune des activités de réseautage, 
de mobilisation et de facilitation envisagées. Ainsi structuré en deux axes transversaux et articulé en chantiers, le Forum peut aspirer à «rendre possible » et à « rendre pertinent » le patrimoine, c'est-à-dire à contribuer à la résolution des problématiques générales auxquels sont inféodés les problèmes spécifiques des interventions et des recherches des membres réunis : le Forum circonscrit en effet une communauté de pratique qui peut à la fois partager les savoirs et permettre aux citoyens d'agir comme des acteurs à part entière de la patrimonialisation. Cette visée définit précisément certaines des activités programmées, qui misent sur la participation discursive afin de permettre aux citoyens, aux décideurs et aux experts de contribuer au débat public sur leur environnement identitaire et de faire avancer l'état des connaissances sur le patrimoine : parmi celles que nous présentons brièvement ci-dessous, le wiki, la conférence télématique et le concours public « J'aime mon église ! » sont représentatives de ce principe participatif déployé dans des véhicules originaux. Puis, par-delà ces activités spécifiques, la visée du Forum de fonder une communauté de pratique repose sur l'intégration des contenus des recherches et des interventions mis en réseau et de la forme de cette mise en réseau des connaissances, des producteurs et des utilisateurs, au sein et à l'extérieur de l'université. L'approche du FCRPP se distinguant en soutenant l'échange entre les producteurs et avec les utilisateurs du patrimoine, la programmation des activités devient dès lors elle-même un instrument de recherche : tandis que le contenu des activités est logé à l'enseigne de l'un ou l'autre des trois thèmes de pointe que désignent les chantiers, la forme des activités doit, elle, contribuer à cette nouvelle compréhension des mécanismes de la patrimonialisation (partant, susciter la réflexion sur l'économie et l'écologie du patrimoine, tout en apportant de nouvelles perspectives de recherche et de pratique).

\section{Activités et médias}

La programmation du FCRPP identifie actuellement vingt-et-une activités-type déployées dans plusieurs médias, allant des ouvrages scientifiques aux quotidiens à grand tirage et du papier à l'Internet. Testées sous forme de pilote, mises sur pied annuellement ou établies en continu de 2008 à 2015, ces activités ciblent soit les chercheurs universitaires, les jeunes chercheurs, les chercheurs gouvernementaux, le milieu associatif, les praticiens (urbanistes, muséologues, etc.), les créateurs (et autres intervenants de la valorisation), les décideurs ou les citoyens, soit - plus généralement - une combinaison de plusieurs de ces producteurs et utilisateurs de connaissances.

Médium central, bilingue, patrimoine-canada.ca débusque et attire un nombre optimal de membres, fédère et diffuse l'information, stimule les communications entre utilisateurs (terminaux ou intermédiaires) et 
producteurs, interagit comme portail (recherche et formation), rend disponibles les données sur le patrimoine (cela inclut la publication en ligne d'un nombre maximal d'écrits et d'activités, le pointage et la mise en commun des banques de données) et soutient les activités d'enseignement et de formation du FCRPP. Surtout, le site vise à susciter et à cimenter la communauté de pratique et tirant parti des possibilités du réseautage par Internet comme source de motivation des participants, motivation d'autant favorisée ici par le géoréférencement des "patrimoines». Afin de stimuler l'engagement interactif et de développer une structure de gouvernance participative du patrimoine, grâce à la réunion des questions des citoyens et des réponses des chercheurs et praticiens (et vice-versa), patrimoine-canada.ca rassemblera, à terme : $1^{\mathrm{o}}$ une conférence télématique, c'est-à-dire ensemble de forums à accès libre ou restreint (voir teluq.uquebec.ca/forums), modérés en fonction des sujets (pédagogie, chantier, etc.), dans lesquels les membres du FCRPP interviendront autour de leurs questions, ou de celles qu'à terme les citoyens leur soumettront. S'intègre ici la recherche sur l'expérience et la communication patrimoniales, qui en retour nourrit l'interaction citoyens/ experts (voir aussi les activités 5, 6, 10, c'est-à-dire les blogues, les concours et les conférences). $2^{\circ}$ Un observatoire, d'abord des églises, puis de la recherche sur le patrimoine, que les membres et l'agrégation conventionnelle de contenus, alimenteront de solutions locales. L'observatoire sera aussi assorti d'un bulletin électronique mensuel axé sur l'application des connaissances et la mise en commun des pratiques. $3^{\circ}$ Un wiki, à destination du public élargi, qui poursuivra deux objectifs. D'une part, la diffusion/consultation des savoirs produits, par objet de recherche, dans le cadre des projets des membres; d'autre part, un Dictionnaire des grandes figures du patrimoine canadien (notices de $\sim 1000$ mots, constitué par appel à textes dans le cadre des congrès partenaires, séminaires de formation et autres), qui affranchira les connaissances des provinces/régions dans lesquelles elles sont emprisonnées. $4^{\circ}$ Aussi pour mettre à niveau les savoirs et favoriser leur redéploiement, une revue, Patrimonium, première revue de recherche sur les phénomènes patrimoniaux et la patrimonialisation. $5^{\circ}$ Blogues et clavardages, sur des thèmes canadiens ou locaux, se grefferont, selon les besoins, pour mettre à profit la toile pancanadienne de chercheurs et de partenaires, de groupes associatifs (diffusion) ou sociétés savantes locales (documentation), au fil notamment de «concours». En effet, le Forum entend rejoindre directement les utilisateurs « terminaux » du patrimoine - les citoyens - pour les informer, stimuler leur participation, mais aussi pour connaître les motifs de leur affection patrimoniale. Si les contenus des activités $2^{\circ}, 3^{\circ}$ et $4^{\circ}$ (observatoire, wiki, revue) alimenteront, en amont, des trousses éducatives, on peut, en aval, envisager un sondage formel ; mais surtout, le Forum permet 
de coupler, entre chercheurs et société civile, sondage et échange, à travers une activité comme $\left(6^{\circ}\right)$ un concours public prenant la forme d'un dialogue itératif dans les médias à grand tirage, par exemple « J'aime mon église ! » qui sollicitera des lecteurs l'exposé de leur «affection » en assortissant la publication de celui-ci d'une analyse « experte » de l'église en question. De façon plus traditionnelle, en partenariat avec les Éditions Multimondes et l'University of Alberta Press, le Forum soutient $7^{\circ}$ les collections « Heritage Studies » et " Architectural History in Canada », en parallèle des " Cahiers de l'Institut du patrimoine » (Multimondes) et de la collection « Patrimoine urbain » (Presses de l'Université du Québec), de même que diverses activités d'édition, comme l'Encyclopédie du patrimoine culturel (www.ameriquefrancaise.org). Ponctuellement, autour des chantiers, la programmation prévoit bien sûr des $\left(8^{\circ}\right)$ articles (anglais ou français), dont ceux de jeunes chercheurs sélectionnés par concours pancanadiens, dans Architecture Canada, Rabaska, Ethnologies, etc., dont le Forum espère accroître la circulation pancanadienne, ainsi que $\left(9^{\circ}\right)$ des numéros thématiques de revues professionnelles (ArQ, Muséologies, Canadian Architect, Plan, etc.). $10^{\circ}$ Conférences publiques, articles de vulgarisation, documents d'interprétation, sessions de formations, etc., continueront d'être produits (chercheurs et partenaires), et leur impact (grand public et formation des chercheurs), soutenu par le Forum.

\section{Formation et formation à la recherche}

Le FCRPP encourage aussi tout particulièrement la participation directe et la mobilité des jeunes chercheurs, que ciblent, hormis divers encouragements, onze des vingt-et-une activités-type programmées de 2008 à 2015. De façon générale, le Forum mise sur la multiplication des perspectives d'accueil pour les postdoctorants et, pour les doctorants, sur la réunion des formations dans des parcours transversaux (entre les programmes) et sur des cotutelles interuniversitaires. Il s'agit de rendre chacun des programmes participants plus attractif et de former un plus grand nombre de chercheurs mieux outillés et « maillés ». Par ailleurs, à mesure qu'on concevra des recherches conjointes, des étudiants se verront attribuer, autour de chantiers spécifiques, des tâches qui leur permettront de fréquenter les différents univers des membres du Forum.

Onzièmement, la programmation du forum compte pour activité-type des stages dans les milieux associatifs du patrimoine et chez d'autres partenaires ; $12^{\circ}$, la mise en place de cadres de cotutelles formelles entre des universités canadiennes, comme celui que l'Institut du patrimoine de l'Université du Québec à Montréal (UQȦM) entretient actuellement pour le Doctorat international en médiation, muséologie et patrimoine, et des parcours 
transversaux, sur la base de l'inventaire des programmes. La formation à la recherche au sein du Forum s'appuie aussi sur les $\left(13^{\circ}\right)$ Rencontres internationales des jeunes chercheurs en patrimoine, la quatrième édition de celles-ci sur « Patrimoine et sacralisation / Patrimonialisation et sacré » étant organisée à l'UQÀM, en septembre 2008. Annuellement aussi, $\left(14^{\circ}\right)$ un séminaire doctoral, La patrimonialisation, convoque des membres à une formation pluridisciplinaire; $15^{\circ}$, un cours pancanadien, notamment à l'enseigne du «patrimoine religieux », éventuellement diffusé sur Internet ou télédiffusé, sera offert aux premier et deuxième cycles universitaires des programmes participants.

Le réseautage, compte tenu entre autres de la diversité des milieux (praticiens, associatifs, institutionnels, gouvernementaux, etc.) mise aussi sur la mobilité géographique des participants. Pour ce, le Forum compte annuellement, en sus de la Rencontre internationale des jeunes chercheurs en patrimoine, sur quatre types de rencontres scientifiques formelles. $16^{\circ} \mathrm{Le}$ « Forum des décideurs » expérimenté en 2006 et renouvelé en 2008, auquel fait écho $\left(17^{\circ}\right)$ la participation à Les arts et la ville et aux rencontres annuelles des partenaires ; $18^{\circ}$, des minicolloques mettant l'accent sur les travaux des jeunes chercheurs et déployés dans le cadre d'un congrès plus large ; $19^{\circ}$, une table ronde annuelle et itinérante (Charlottetown en 2006, Yellowknife en $2008 \ldots$..) des membres ; $20^{\circ}$, un colloque national ou international annuel, notamment pour élargir notre communauté et ses impacts, comme celui tenu en 2006 à l'Université Sainte-Anne ( Le Patrimoine religieux de la NouvelleÉcosse »), celui organisé en 2009 par l'UQÀM, l’Université Concordia, l'Université Laval et l'Université de Montréal sur « Des couvents en héritage / Religious Houses. A Legacy » et celui envisagé, à l'University of British Columbia, sur « The Narratives of Heritage ». À cela s'ajoute $\left(21^{\circ}\right)$ une activité couplant formation des professionnels et maillage (décideurs, créateurs et autres praticiens) dans le cadre d'une École d'été du patrimoine (voir par exemple, www.arc.ulaval.ca/futursetudiants/insitu).

\section{Chercheurs, collaborateurs et partenaires}

L'Institut du patrimoine de l'UQÀM agit comme tête de pont du FCRPP. Luc Noppen, titulaire de la Chaire de recherche du Canada en patrimoine urbain, coordonne le Forum; il en dirige aussi le chantier sur le «patrimoine religieux ", tandis que Lucie K. Morisset et France Vanlaethem dirigent, respectivement, le chantier "mémoire patrimoniale» et le chantier « patrimoine moderne ». Outre ces trois chercheurs et professeurs de l'UQÀM, le Forum, selon sa configuration avalisée par le Conseil de recherche en sciences humaines du Canada, regroupe neuf chercheurs principaux et six chercheurs collaborateurs. Il s'agit de : Yves Bergeron (UQÀM), Martin Cherry 
(University of Bath), Thomas Coomans de Brachène (Vrije Universiteit Brusseul), Martin Drouin (UQÀM), Philippe Dubé (Université Laval, Québec), Denis Gagnon (Collège de Saint-Boniface), Jean-Michel Leniaud (École pratique des hautes études, Paris), Richard MacKinnon (Cape Breton University), Tania Martin (Université Laval, Québec), Jean-Pierre Pichette (Université Sainte-Anne), Robert Shipley (University of Waterloo), Geoffrey Simmins (University of Calgary), Laurier Turgeon (Université Laval, Québec) et Rhodri Windsor-Liscombe (University of British Columbia, Vancouver). Les onze organes partenaires du Forum sont : les Éditions multimondes, le Conseil du patrimoine religieux du Québec, Heritage Canada, Heritage Foundation of Newfoundland and Labrador, Inuit Heritage Trust, le Laboratoire de muséologie et d'ingénierie de la culture, Les arts et la ville, Ontario Heritage, les Presses de l'Université du Québec, la Société pour l'étude de l'architecture au Canada et l'Université Concordia. 